\title{
The development of green wireless mesh network: A survey
}

\author{
Yuan Chai, Xiao-Jun Zeng \\ Department of Computer Science, University of Manchester, Manchester M13 9PL, UK.
}

Correspondence to: Prof. Xiao-Jun Zeng, Department of Computer Science, University of Manchester, Oxford Road, Manchester M13 9PL, UK. E-mail: x.zeng@manchester.ac.uk

How to cite this article: Chai Y, Zeng XJ. The development of green wireless mesh network: A survey. J Smart Environ Green Comput 2021;1:47-59. https://dx.doi.org/10.20517/jsegc.2020.05

Received: 30 Dec 2020 First Decision: 02 Mar 2021 Revised: 07 Mar 2021 Accepted: 11 Mar 2021 Available online: 30 Mar 2021

Academic Editors: Witold Pedrycz, Patricia Melin Copy Editor: Xi-Jun Chen Production Editor: Xi-Jun Chen

\begin{abstract}
Wireless mesh network (WMN) is a type of self-healing, self-configuration, and peer-to-peer wireless network. Without expensive and fixed base stations, WMN can be established fast, easily, and flexibly at a low cost. The convenience and flexibility of establishing WMN brings a wide range of applications. The request of high bandwidth, high coverage and high transmission rate can be satisfied. Current research on WMN involves routing, resource allocation, mobility control, security and so on. With the increasing concern of green communication, improving energy efficiency is more and more important nowadays. As energy harvesting can overcome energy constraints and extend the network lifetime, it has attracted the great attention from many researchers when designing network. Further, WMN is an important networking mode in next-generation communication to guarantee the quality of service and reduce the networking complexity. Due to such importance, a survey of the research and development in WMN including the future research direction and opportunities is given in this paper.
\end{abstract}

Keywords: Green wireless mesh network, energy saving, routing, resource allocation, mobility control, security, machine learning, software-defined networking

\section{INTRODUCTION}

Wireless mesh network (WMN) is a low-cost network without fixed base stations, and it is self-healing with high robustness. Due to the mesh networking mode, when a mesh node is failed or broken to forward data, 
alternative nodes and paths will be discovered to guarantee the communication. The single point of failure problem will be solved. WMN consists of mesh routers and mesh clients. Mesh routers always have multiple interfaces, with more energy capacity, while mesh clients often have only one interface, with very limited energy. The mobility of mesh clients is higher than that of mesh routers. For example, smartphones, laptops, unmanned aerial vehicles and so on can be mesh clients, and domestic access routers can be mesh routers ${ }^{[1]}$. $\mathrm{WMN}$ is categorized into 3 types: infrastructure $\mathrm{WMN}$, client $\mathrm{WMN}$ and hybrid $\mathrm{WMN}^{[2]}$ :

- Mesh routers compose the infrastructure WMN. In infrastructure WMN, mesh clients can only access mesh routers by one hop to complete communication.

- Client WMN is very similar to the pure ad hoc network, which is composed by mesh clients. In this case, mesh clients can communicate directly with each other without accessing mesh routers.

- Hybrid WMN is combined by infrastructure WMN and client WMN. With the most flexible networking mode, hybrid WMN has the potential to be applied in many different scenes.

WMN can make heterogeneous devices cooperate with each other. Different types of networks like cellular network, WiMAX, vehicular network, Internet of things, sensor network and so on can be cooperative with each other through WMN. The access points of different networks can become the mesh routers of WMN. The data traffic can be from a mesh node to the Internet through the gateway or from a mesh node to another mesh node. The traffic flows and network topology are very flexible to satisfy the communication requests. Most traffic flows from source to destination need to be forwarded through multiple hops. The coverage will be extended to make it possible for remote nodes to access the Internet without increasing the transmission power ${ }^{[3]}$. However, interference will be caused by the feature of multiple hops. Multi-hop communication brings more complicated relationships among mesh nodes. The links using the same wireless channel within the interference range will be interfered mutually. Especially in next-generation wireless communication, the number of devices will increase dramatically and the network density will be very high. Therefore, the network performance decrease like packet loss resulting from interference should be considered and addressed carefully ${ }^{[4]}$. The wireless resources like channels and spectrum need to be used sufficiently and effectively. The objective of network deployment is to transmit most data by using the least resource.

In addition to the wireless resources, energy is another important aspect and resource which should be consumed efficiently. With the development of green communication, saving energy has become a more and more important and critical issue ${ }^{[5]}$. Some devices like sensors and smartphones have very limited energy. Once the energy is run out of, the device cannot be used anymore and it will become a dead node. With more and more dead nodes appearing, the connection of network will decrease and some nodes may even become isolated nodes. To avoid this circumstance, energy should be consumed in balance. Further, energy harvesting $(\mathrm{EH})$ which can overcome energy constraints has attracted many researchers' attention these years ${ }^{[6]}$. Mesh nodes can obtain energy from solar, wind, radio frequency signals and so on. With the ability of obtaining and saving energy, network lifetime can be extended.

As WMN is flexible with low cost, it is an important networking mode in next-generation wireless communication. It can be established in some rural areas where it is hard to build expensive base stations. In addition, WMN can also be used in military and emergency communication due to the flexible topology. In Internet of things, many devices have a very limited transmission range, so WMN can help complete communication by multiple hops. As WMN plays an important role in wireless communication, it is 
essential to deploy WMN efficiently by considering its characteristics. The network deployment which can improve network performance includes many research areas like routing, resource allocation, mobility control, security, energy saving, etc. The detailed recent research is given in Section 2 below. Then the future research opportunities and technologies are provided in Section 3. Conclusions and challenges are summarized in Section 4.

\section{CURRENT RESEARCH IN WMN}

Current research in WMN focuses on different aspects. In the development process of WMN, routing, resource allocation, mobility control, and security are important areas all the time. Recently, energy saving has become a more and more heated topic with the development of green communication. Detailed works in WMN are given as follows.

\section{Routing design}

Routing is always one of the most important aspects in network design because different route selections will bring different network performance. According to the route establishing time, routing can be categorized into proactive ${ }^{[7]}$, reactive ${ }^{[8]}$ and hybrid routing ${ }^{[9]}$. Proactive routing methods actively discover and maintain routes all the time. When there is a communication request, less responding delay will be obtained because routes are already known. However, the control overhead is large. Reactive routing methods only start to find routes when nodes need to start communication and transmit packets. The control overhead is less as control packets do not need to be transmitted all the time. Hybrid routing combines the proactive and reactive routing, and different routing methods can be used among different types of nodes. According to the features of mesh routers and mesh clients, proactive routing is suitable for static mesh routers and reactive routing can work well for mobile mesh clients.

The proactive routing can be formulated as an optimization problem, which can be addressed and supported by software-defined networking (SDN) technology with global network view ${ }^{[10]}$. The objective is the metric to evaluate the performance of routes. Considering different requests of Quality of Service (QoS), different aspects like Expected Transmission Time (ETT ${ }^{[11]}$, Expected Transmission Count (ETX ${ }^{[12]}$, throughput ${ }^{[13]}$, packet error ratio ${ }^{[14]}$, energy consumption ${ }^{[15]}$, hop count ${ }^{[16]}$ and so on can be considered. When solving the established routing optimization problem, genetic algorithm $(\mathrm{GA})^{[11]}$, particle swarm optimization $^{[17]}$, multi-objective differential evolution $(\mathrm{MODE})^{[18]}$, ant colony optimization $(\mathrm{ACO})^{[19]}$, reinforcement learning ${ }^{[20,21]}$, fuzzy logic ${ }^{[22,23]}$, etc. can be used.

To adapt to the features of mobile mesh clients, most designed routing methods for WMN are reactive routing. When design routing metric, factors which will influence network performance like delay ${ }^{[24,25]}$, load $^{[26,27]}$, congestion ${ }^{[28]}$, interference ${ }^{[4,29]}$, mobility ${ }^{[30]}$, security ${ }^{[1,32]}$, stability ${ }^{[33]}$, and energy ${ }^{[34]}$ can be considered. As the factors can influence one another, more than one factor like delay, intra- and inter-flow interference can be considered at the same time ${ }^{[35]}$. When the delay cannot meet the threshold request, transmission rate and power will be reduced. ETX and ETT are often used as the basis of many researches. In addition to ETX, available bandwidth and channel diversity are considered in ${ }^{[25]}$. With larger retransmission times, the energy consumption will be higher ${ }^{[36]}$. Besides ETT $^{[27]}$, considers load condition and interference. For the routing mechanism, some research considers the flooding control of control packets. AODV-DF ${ }^{[37]}$ reduces the number of route request (RREQ) packets and controls the flooding effectively. More wireless resources can be used to serve the data packets rather than control packets, and load will be reduced. 
However, the research of hybrid routing is much less. $\mathrm{HMesh}^{[38]}$ combines the proactive routing protocol $\mathrm{OLSR}^{[39]}$ and reactive routing protocol $\mathrm{AODV}^{[40]}$. OLSR is used among mesh routers, and AODV is used among mesh clients. $\mathrm{HDV}^{[41]}$ uses tree-based proactive routing between mesh routers and gateway, to set reactive routing among mesh clients. Hop count is the only factor considered in routing metric of both HMesh and HDV. CHRP ${ }^{[42]}$ improves the routing metric, considering interference, channel condition and energy of mesh clients. The access process is neglected, and the mesh router which can response route reply (RREP) to the mesh client will be accessed unconditionally. To consider the load condition of the access mesh routers, $\mathrm{LA}-\mathrm{CHRP}^{[43]}$ selects the mesh router with less load as the access node. However, the condition of the whole proactive path and the regional condition are neglected. RCA-HRP ${ }^{[44]}$ considers and evaluates the condition of whole proactive path. In addition, the regional information like load, speed and energy is considered.

\section{Resource allocation}

As wireless resource is limited, effective resource allocation is very important. Channel allocation ${ }^{[45,46]}$ and rate allocation ${ }^{[4]}$ can maximize network throughput and fairness. The relationship between topology and the available number of channels which can be assigned is given $\mathrm{in}^{[45]}$. Different factors and network design areas jointly influence network performance, so many works jointly consider different areas as a whole. Channel assignment and routing can be designed jointly ${ }^{[48]}$. Routing is designed after channel allocation. The derived outage probability is used to determine the constraint of energy consumption. When considering routing, channel allocation and rate scheduling at the same time, Lagrange duality method can be used to convert the corresponding convex optimal solution into 3 simple sub-problems ${ }^{[49]}$. Channel allocation and scheduling can be implemented cooperatively ${ }^{[50]}$. When channels are allocated, loss, interference and channel busy level are considered. In scheduling of sending interface, longer switch interval is given for busy interface with long queue. Jointly allocating resources according to different requests can bring better whole network performance.

With the support of edge computing, more computing devices and resources are provided. As a result, the burden and load via the distributed cloud are then reduced, and the response time can be significantly shortened. WMN can be deployed to bring a new paradigm to enable distributed intelligence ${ }^{[51]}$, and the research on resource and task allocation is more and more heated ${ }^{[52]}$. Computing capacity, computing delay and transmission delay should be considered to select the best place to offload tasks ${ }^{\left[{ }^{33]}\right.}$.

\section{Mobility control}

Mobility will bring flexible and changeable network topology, which will influence network performance. To reproduce the movement features is the main purpose of the mobility model ${ }^{[54]}$. Clustering and region division are effective to control mobility and distinguish features of different nodes ${ }^{[20]}$. Then traffic transmission can be completed based on clusters. A cluster-based network has better scalability, and nodes will be classified into cluster heads, cluster members and gateways. The technology of clustering makes the deployment of large-scale WMN possible ${ }^{[55]}$. The nodes with similar mobility features can be classified into one cluster because their neighborhood relationship is relatively stable. Therefore, clusters do not need to be established frequently, which guarantees the stable communication. After being clustered, the cost of maintaining the network condition is reduced because the gateways only need to maintain the information of cluster heads and the condition of cluster members is monitored by cluster heads.

With the fast development of wireless mobile communication, more and more mobile devices need to access the Internet. As a result, the density of a network is high, and it will be more and more difficult to control the network. Clustering is also a good way to serve the traffic in dense network ${ }^{[56]}$. To save the energy of mobile devices, the node with enough energy can be selected as the cluster head to help support 
the communication among cluster members. The cluster heads can also be selected according to the load, capacity, location and node degree $\mathrm{e}^{[57-59]}$.

\section{Security}

There are different attacks on different Open System Interconnection layers. To avoid and reduce the impact of attacks on the network performance, intrusion prevention, detection and response mechanisms should be designed according to service requests ${ }^{[0]}$. At physical layer, collision jamming and device tampering may occur. At Medium Access Control layer, passive eavesdropping and jamming attack will decrease the traffic transmission rate. At network layer, black hole, gray hole, worm hole attacks ${ }^{[6]}$, flooding attacks $^{[62]}$, and replay attacks ${ }^{[63]}$ can bring failed data transmission. Flooding and desynchronization attacks can occur at transport layer. The application layer has security risks including logic errors and buffer overflows. The security threats can be generally classified into passive and active attacks. In passive attack, network operation will not be disrupted because the attackers monitor and capture data without triggering real actions. However, the passive attack can be a preparation for active attack. After monitoring and analyzing the network traffic data, the active attack can be done to control and disrupt the normal network operation. The attackers will try to inject, delete or alter data, so the successful transmission rate and network performance will decrease.

It is essential to detect and prevent the damage of attacks on the network. Attacks can be detected by calculating neighbor list and directional neighbor list of the source node ${ }^{[64]}$. Then the location of the attack and influenced nodes can be provided. Reputation model based on forwarding success and failure rates can also evaluate the nodes' behavior and it is beneficial to study the social relationship among nodes. Then groups with similar status are established. The nodes with weak social relationship may be the malicious nodes and they should be avoided being used to forward traffic ${ }^{[65]}$. The trust of nodes can also be calculated by using probability theory and packets can be routed according to fuzzy set theory ${ }^{[66]}$. The network factors like hop count, reliability and link capacity can be considered.

\section{Energy saving}

With the development of green computing and green communication, saving energy is an urgent duty to be completed nowadays, so it has become an emergent and important research topic in WMN recently. Energy should be carefully considered when implementing network design. The mode switch between sleep and active can help save energy ${ }^{[67]}$. To save energy, minimizing the energy consumption ${ }^{[68]}$ or maximizing lifetime ${ }^{[6,70]}$ can be the objective, and EH can be used to obtain energy. Energy will be consumed at different states: monitoring, processing, communication, and state transferring ${ }^{[71]}$. Four actions that are keeping sleeping, turning on collection, turning on processing and turning on transmission can be selected ${ }^{[72]}$. Interfaces can be turned on or off according to the energy and selected paths $\mathrm{in}^{[48]}$. When the obtained energy is more than consumed energy, the energy will be saved. Otherwise, the battery energy will be consumed. Besides energy, other factors like delay ${ }^{[73]}$, throughput ${ }^{[72]}$ and transmission rate $^{[77]}$ can also be considered together. The flooding control can be done to change the probability of forwarding control packets like RREQ to save energy ${ }^{[69,75]}$. When designing routing, few works consider EH. DEARER ${ }^{[74]}$ proposes a cluster-based routing to select cluster head effectively. Energy efficiency and transmission rate are considered, but load and interference are overlooked. The method in ${ }^{[76]}$ minimizes delay, and considers power allocation, tree-based routing and scheduling, which solves the near-far problem. However, interference is still not considered, and it only uses the free-space loss model to describe large scale fading.

Power allocation is another important part which can influence energy condition directly, and it can be done according to the user distribution and predictive $\mathrm{EH}$ model ${ }^{[77]}$. To predict user density in future, the model based on Markov chain can be used. In addition, Gaussian distribution model is used to predict 
harvested energy. Power will then be allocated according to the energy level. Power can also be jointly assigned with routing and spectrum allocation by mixed integer linear programming (MILP) ${ }^{[78]}$.

In short, the summary of representative studies for $\mathrm{WMN}$ is given in Table 1.

\section{OPPORTUNITIES AND TECHNOLOGIES IN FUTURE RESEARCH}

With the features of flexibility, low cost, scalability, multiple hops and so on, WMN will also play an important role in next-generation communication. With the fast grow and development of Internet of Things (IoT), WMN can well integrate with and enhance Io ${ }^{[79]}$. The coverage will be extended and energy will be saved by the support of WMN. With the increasing number of devices, the size and density of WMN will increase. Network deployment needs more intelligent methods to adapt to the complex network conditions. Machine learning (ML) and SDN can bring more opportunities and potentials for WMN.

\section{MACHINE LEARNING FOR SMART WMN}

ML is a heated technology which has been successfully used in many areas like image processing, text mining, natural language processing, agent systems, etc. The intelligence of ML makes it also adaptive to implement network design for $\mathrm{WMN}^{[80]}$.

It is important and beneficial to understand and predict network conditions, which can be done effectively and accurately by ML. With the predicted outcomes, some adjustments can be implemented to adapt to the varying network conditions. The batch ML needs large amounts of data, and cannot be updated quickly and efficiently after training. Therefore, online or real-time ML which requests less data processing at a time can be used to predict link quality with less computing cost and time ${ }^{[8]}$. When multiple bands and channels are available at relay nodes, it is essential to select the one with best quality. The channel quality can be predicted by ML and the smarter channel selection methods based on the ML outcomes can be developed to enable larger network throughput and shorter delay ${ }^{[3,82]}$.

Besides prediction, ML can also be used in routing, resource allocation and strategy deployment, and reinforcement learning like Q learning is a very effective method to suit flexible network without a known transition model ${ }^{[80]}$. It can also solve multi-objective problems for convex or non-convex problems ${ }^{[83]}$. Reward function is set according to the QoS request like load, transmission rate, delay, distance, mobility and energy ${ }^{[84-87]}$. The transmission rate can then be adjusted based on the network capacity and quality of experience ${ }^{[88]}$. The tradeoff between exploitation and exploration needs to be balanced. The exploitation is to use the current best solution, and the exploration is to find more potential and future best solutions. The problem may converge to local optimal solution if exploitation is too focused. Otherwise, the problem cannot converge if exploration is too focused. $\varepsilon$-greedy and soft-max methods are often used to balance exploitation and exploration. Deep learning is also an effective way to learn and control network in real time. Traditional routing protocols may even be removed and replaced by deep reinforcement or other selflearning technologies in future ${ }^{[89]}$. Past mistakes causing congestion and network performance decrease will be learnt to avoid the same mistakes in future. The structures like convolutional neural network ${ }^{[89]}$ and deep belief architecture ${ }^{[90]}$ can be used. The combination of reinforcement learning and deep learning can make use of the advantages of them together. Results and selections can be achieved automatically in any largescale network with a lot of states and actions. Real-time traffic will be controlled efficiently ${ }^{[91]}$. Non-linear, complex and multiple-dimension problems can be solved successfully ${ }^{[92]}$.

Further, with the help of ML, the effects and uncertainties of human element and idiosyncrasy on the development of green WMN can be reduced. The human behavior is not entirely rational and relies on 
Table 1. Review of representative studies for WMN

\begin{tabular}{|c|c|c|c|c|c|c|c|c|c|c|c|}
\hline \multirow{3}{*}{ Ref. } & \multicolumn{9}{|c|}{ Considering aspects } & \multirow{3}{*}{$\begin{array}{l}\text { Network performance } \\
\text { factors }\end{array}$} & \multirow{3}{*}{ Methods } \\
\hline & \multirow[b]{2}{*}{ Routing } & \multicolumn{5}{|c|}{ Resource allocation } & \multirow[b]{2}{*}{ Mobility } & \multirow{2}{*}{$\begin{array}{l}\text { Energy } \\
\text { saving }\end{array}$} & \multirow{2}{*}{ Security } & & \\
\hline & & $\begin{array}{l}\text { Channel } \\
\text { allocation }\end{array}$ & Scheduling & $\begin{array}{l}\text { Rate } \\
\text { adaptation }\end{array}$ & $\begin{array}{l}\text { Power } \\
\text { control }\end{array}$ & $\begin{array}{l}\text { Task } \\
\text { allocation }\end{array}$ & & & & & \\
\hline Mahmood et al. ${ }^{[11]}$ & $\sqrt{ }$ & & & & & & & & & ETT, hop count & $\mathrm{GA}$ \\
\hline Murugeswari et al. ${ }^{[12]}$ & $\sqrt{ }$ & & & & & & & & & ETX, delay & $\begin{array}{l}\text { Multi-objective } \\
\text { evolutionary algorithm }\end{array}$ \\
\hline Bokhari et al. ${ }^{[19]}$ & $\sqrt{ }$ & & & & & & & & & Interference, delay & $\mathrm{ACO}$ \\
\hline Yadav et al. ${ }^{[22]}$ & $\sqrt{ }$ & & & & & & $\sqrt{ }$ & $\sqrt{ }$ & & Delay, bandwidth & Fuzzy logic \\
\hline Chai et al. ${ }^{[43]}$ & $\sqrt{ }$ & & & & & & & $\sqrt{ }$ & & Load, energy & Hybrid routing \\
\hline Avallone et al. ${ }^{[48]}$ & $\sqrt{ }$ & $\sqrt{ }$ & & & & & & $\sqrt{ }$ & & Energy, outage probability & $\begin{array}{l}\text { Heuristic, linear } \\
\text { programming }\end{array}$ \\
\hline Deng et al. ${ }^{[50]}$ & & $\sqrt{ }$ & $\sqrt{ }$ & & & & & & & Throughput, load, delay & Huffman tree \\
\hline Suto et al. ${ }^{[77]}$ & & & & & $\sqrt{ }$ & & & $\sqrt{ }$ & & User distribution & Markov chain prediction \\
\hline Liu et al. ${ }^{[52]}$ & & & & & & $\sqrt{ }$ & & & & $\begin{array}{l}\text { Delay, cost (computing, storage, } \\
\text { data transmission) }\end{array}$ & $\begin{array}{l}\text { Prediction-based } \\
\text { online algorithm }\end{array}$ \\
\hline Tang et al. ${ }^{[47]}$ & & $\sqrt{ }$ & & $\sqrt{ }$ & & & & & & Throughput, fairness & $\begin{array}{l}\text { Linear programming, } \\
\text { convex optimization }\end{array}$ \\
\hline Ramakrishnan et al. $^{[54]}$ & $\sqrt{ }$ & & & & & & $\sqrt{ }$ & & & Throughput & Clustering \\
\hline Li et al. ${ }^{[20]}$ & $\sqrt{ }$ & & & & & & $\sqrt{ }$ & & & Delay, hop count, delivery ratio & Reinforcement learning \\
\hline Kassan et al. ${ }^{[71]}$ & & & & & & & & $\sqrt{ }$ & & Packet forwarding & $\begin{array}{l}\text { Game theory, clustering, } \\
\text { EH }\end{array}$ \\
\hline Maleki et al. ${ }^{[73]}$ & $\sqrt{ }$ & & & & & & & $\sqrt{ }$ & & Delay, energy, channel condition & Reinforcement learning \\
\hline Dong et al. ${ }^{[74]}$ & $\sqrt{ }$ & & & & & & & $\sqrt{ }$ & & Distance, energy, channel & Clustering \\
\hline Yao et al. ${ }^{[76]}$ & $\sqrt{ }$ & & $\sqrt{ }$ & & $\sqrt{ }$ & & & & & $\begin{array}{l}\text { Node location, delay, fading, } \\
\text { load }\end{array}$ & $\begin{array}{l}\text { EH, Markov decision } \\
\text { process }\end{array}$ \\
\hline Yu et al. ${ }^{[65]}$ & $\sqrt{ }$ & & & & & & & & $\sqrt{ }$ & Neighborhood relationship & Reputation model \\
\hline Pugalendhi et al. ${ }^{[66]}$ & $\sqrt{ }$ & & & & & & & & $\sqrt{ }$ & Packet transmission ratio & $\begin{array}{l}\text { Probability theory, fuzzy } \\
\text { theory }\end{array}$ \\
\hline
\end{tabular}

ETT: Expected transmission time; ETX: expected transmission count; GA: genetic algorithm; ACO: ant colony optimization; EH: energy harvesting.

existing rules and experiences to large extent. Due to the complicated conditions and potential states, it is really hard for human to give best and secure solutions according to the known knowledge. The results, therefore, may not be the expected ones by the engineering design perspective. Therefore, the influence of unreasonable human behavior should be avoided as far as possible. ML can learn from data and implement automatic adaptation, so the 
uncertainties in human decisions can be reduced by taking advantage of ML. Further evaluation of ML in the real world should be implemented to confirm the effectiveness and usefulness of ML in WMN ${ }^{[93]}$.

\section{Software-defined networking for effective WMN}

SDN, which is an approach to network management that enables dynamic, programmatically efficient network configuration, is also an essential technology in next-generation communication with the capability of decoupling data and control planes. The programmability is improved and it will be easy to manage the whole network ${ }^{[94]}$. SDN can be applied in existing WMN to combine the advantages of centralized control in $\mathrm{SDN}$ and low cost in $\mathrm{WMN}^{[95-97]}$. The centralized network controller can provide high computing ability and storage capability. The whole network information can be obtained by the network controller from switches, and strategies are made according to the information ${ }^{[98]}$. The rules of routing or resource control computed by the controller will be transferred and installed to the nodes in network ${ }^{[99]}$. The switches can be simpler because they do not need to make decisions anymore and they can just forward data based on the rules computed by the network controller.

Better mobility management, routing and resource allocation can be achieved. The combination of SDN and $\mathrm{WMN}$ has better performance than pure $\mathrm{WMN}^{[100]}$. Service requests can be satisfied by simply updating software rather than changing hardware. Current research about SDN in WMN is still rather limited, and the experiments in real platforms are still needed in the future. Besides, SDN and ML can be combined together to make the network more intelligent.

\section{CONCLUSIONS AND CHALLENGES}

WMN is an important networking mode which has been researched and developed for many years. It will still play an important role in next-generation communication due to its multi-hop, flexible and low-cost features. Current research on WMN including routing, resource allocation, mobility control, security and energy saving is summarized in this survey. To improve the whole network performance, further research works need to consider different aspects together. To provide the smarter and more effective services in the future, ML and SDN can be integrated with WMN to realize such a goal. ML can intelligently predict network conditions and learn from the environment to find best solutions and avoid mistakes. Besides, the ability of self-adaptation can bring more intelligence and reduce the influence of human behavior on the development of green WMN. SDN makes the network management more flexible, and more complicated tasks can be completed by the network controller.

Devices deployment will be more and more flexible in the future. Any device can join, leave and move at any time, which brings very flexible topology. Topology deployment and node placement will become a challenging area. Different placements will bring different neighborhood and interference relationships. When designing the placement, the number of nodes connected to the access point should be controlled to avoid high congestion. In addition, when there are multiple gateways in the network, the gateway placement and selection are very important for balancing load. The node placement problem can be formulated as a multi-objective problem to satisfy delay, transmission rate, fairness and energy ${ }^{[101,102]}$. In big-data era, the locations of services are also very important to reduce electricity $\cos ^{[103]}{ }^{[0}$ The topology design of network, services, computing resources, etc. are all important for serving traffic with QoS requests.

When evaluating the performance of different ideas and schemes, it should be kept in mind that the environment in the real world is different from simulation platforms. Thus, it is essential to evaluate network performance by experiments in the real world ${ }^{[104]}$ in the future. 


\section{DECLARATIONS}

\section{Authors' contributions}

Made substantial contributions to methodology, summarization and writing: Chai $Y$

Provided insightful directions, supervision and did proofreading: Zeng XJ

\section{Availability of data and materials}

Not applicable.

\section{Financial support and sponsorship}

None.

\section{Conflicts of interest}

Both authors declared that there are no conflicts of interest.

\section{Ethical approval and consent to participate}

Not applicable.

\section{Consent for publication}

Not applicable.

\section{Copyright}

(c) The Author(s) 2021.

\section{REFERENCES}

1. Akyildiz IF, Wang X, Wang W. Wireless mesh networks: a survey. Comput Netw 2005;47:445-87. DOI

2. Benyamina D, Hafid A, Gendreau M. Wireless mesh networks design-A survey. IEEE Commun Surv Tutor 2011;14:299-310. DOI

3. Sakib S, Tazrin T, Fouda MM, Fadlullah ZM, Nasser N. An efficient and light-weight predictive channel assignment scheme for multi-band B5G enabled massive IoT: A deep learning approach. IEEE Internet Things J 2020:136-41. DOI

4. Boushaba M, Hafid A, Gendreau M. Source-based routing in wireless mesh networks. IEEE Syst J 2014;10:262-70. DOI

5. Wang X, Vasilakos AV, Chen M, Liu Y, Kwon TT. A survey of green mobile networks: Opportunities and challenges. Mob Netw Appl 2012;17:4-20. DOI

6. Ulukus S, Yener A, Erkip E, et al. Energy harvesting wireless communications: A review of recent advances. IEEE J Sel Areas Commun 2015;33:360-81. DOI

7. Kaur R, Rai MK. A novel review on routing protocols in MANETs. Undergraduate Academic Research Journal (UARJ) 2012;1:1038 .

8. Bachega SJ, Tavares DM. Simulation of reactive routing protocols in wireless mesh networks: a systematic literature review. 2014. Available from: https://www.semanticscholar.org/paper/Simulation-of-Reactive-Routing-Protocols-in-Mesh-\%3A-BachegaTavares/ab4c9f1089552a45b192a2874bc3f46014322937. [Last accessed on 19 Mar 2021].

9. Pirzada AA, Portmann M, Wishart R, Indulska J. SafeMesh: A wireless mesh network routing protocol for incident area communications. Pervasive Mobile Comput 2009;5:201-21. DOI

10. Sajjadi D, Tanha M, Pan J. A comparative study of channel switching latency for conventional and SDN-based routing in multi-hop multi-radio wireless mesh networks. Proceedings of the 13th IEEE Annual Consumer Communications \& Networking Conference; 2016 Jan 9-21; Las Vegas, NV, USA. New York: IEEE; 2016. p. 330-4. DOI

11. Mahmood K, Nazir B, Khan IA, Shah N. Search-based routing in wireless mesh network. EURASIP J Wirel Commun Netw 2017. DOI

12. Murugeswari R, Radhakrishnan S, Devaraj D. A multi-objective evolutionary algorithm based QoS routing in wireless mesh networks. Applied Soft Computing 2016;40:517-25. DOI

13. Alotaibi E, Ramamurthi V, Batayneh M, Mukherjee B. Interference-aware routing for multi-hop wireless mesh networks. Comput Commun 2010;33:1961-71. DOI

14. Liu CH, Gkelias A, Leung KK. A cross-layer framework of QoS routing and distributed scheduling for mesh networks. Proceedings of VTC Spring 2008-IEEE Vehicular Technology Conference; 2008 May 11-14; Marina Bay, Singapore. New York: IEEE; 2008. p. 2193-7. DOI

15. Rahat AAAM, Everson RM, Fieldsend JE. Multi-objective routing optimisation for battery-powered wireless sensor mesh networks. GECCO '14: Proceedings of 2014 Annual Conference on Genetic and Evolutionary Computation; Vancouver, BC, Canada. New York: Association for Computing Machinery; 2014. p. 1175-82. DOI

16. Lozano-Garzon C, Camelo M, Vila P, Donoso Y. A multi-objective routing algorithm for wireless mesh network in a smart cities 
environment. Journal of Networks 2015;10:60. DOI

17. Hafid A, Hallam N, Gendreau, M, Maureira JC. A hybrid nature-inspired optimizer for wireless mesh networks design. Comput Commun 2012;35:1231-46. DOI

18. Murugeswari R, Radhakrishnan S. Discrete multi-objective differential evolution algorithm for routing in wireless mesh network. Soft Computing 2016;20:3687-98. DOI

19. Bokhari F, Zaruba G. On the use of smart ants for efficient routing in Wireless Mesh Networks. Available from: https://arxiv.org/abs/1209.0550. [Last accessed on 22 Mar 2021].

20. Li F, Song X, Chen H, Li X, Wang Y. Hierarchical routing for vehicular ad hoc networks via reinforcement learning. IEEE Trans Veh Technol 2018;68:1852-65. DOI

21. Ghaffari A. Real-time routing algorithm for mobile ad hoc networks using reinforcement learning and heuristic algorithms. Wireless Netw 2017;23:703-14. DOI

22. Yadav AK, Das SK, Tripathi S. EFMMRP: Design of efficient fuzzy based multi-constraint multicast routing protocol for wireless ad-hoc network. Computer Networks 2017;118:15-23. DOI

23. Das SK, Tripathi S. Intelligent energy-aware efficient routing for MANET. Wireless Netw 2018;24:1139-59. DOI

24. Li H, Cheng Y, Zhou C, Zhuang W. Minimizing end-to-end delay: A novel routing metric for multi-radio wireless mesh networks. Proceedings of IEEE INFOCOM 2009; 2009 Apr 19-25; Rio de Janeiro, Brazil. New York: IEEE; 2009. p. 46-54. DOI

25. Zhou H, Huang C, Cheng Y, Wang G. A new multi-metric QoS routing protocol in wireless mesh network. Proceedings of 2009 International Conference on Networks Security, Wireless Communications and Trusted Computing; 2009 Apr 25-26; Wuhan, China. New York: IEEE; 2009. p. 459-67. DOI

26. Ali RF, Kiani AK, Pirzada AA. Load dependent dynamic path selection in multi-radio hybrid wireless mesh networks. Proceedings of 2014 IEEE Wireless Communications and Networking Conference (WCNC); 2014 Apr 6-9; Istanbul, Turkey. New York: IEEE; 2014. p. 2020-5. DOI

27. Hao Z, Li Y. An adaptive load-aware routing algorithm for multi-interface wireless mesh networks. Wireless Netw 2015;21:557-64. DOI

28. Khasawneh FA, Kadoch M. Adaptive transmission rate congestion aware routing algorithm in wireless mesh network. Wireless Pers Commun 2017;97:4327-42. DOI

29. Pirzada AA, Wishart R, Portmann M. Congestion aware routing in hybrid wireless mesh networks. Proceedings of the 15th IEEE International Conference on Networks; 2007 Nov 19-21; Adelaide, SA, Australia. New York: IEEE; 2007. p. 513-8. DOI

30. Song W, Fang X. Cross-layer routing with link quality and stability-aware in ITS hybrid wireless mesh networks. Proceedings of the 6th International Conference on Advanced Language Processing and Web Information Technology (ALPIT 2007); 2007 Aug 22-24; Luoyang, China. New York: IEEE; 2007. p. 304-8. DOI

31. Khan S, Loo J. Cross layer secure and resource-aware on-demand routing protocol for hybrid wireless mesh networks. Wireless Pers Commun 2012;62:201-14. DOI

32. Paris S, Nita-rotaru C, Martignon F, Capone A. Cross-layer metrics for reliable routing in wireless mesh networks. IEEE/ACM Trans Networking 2013;21:1003-16. DOI

33. Boushaba M, Hafid A, Gendreau M. Node stability-based routing in wireless mesh networks. J Netw Comput Appl 2017;93:1-12. DOI

34. Kiani AK, Ali RF, Rashid U. Energy-load aware routing metric for hybrid wireless mesh networks. Proceedings of the 2015 IEEE 81st Vehicular Technology Conference (VTC Spring); 2015 May11-14; Glasgow, UK. New York: IEEE; 2015. p. 1-5. DOI

35. Narayan D, Mudenagudi U. A cross-layer framework for joint routing and rate adaptation in infrastructure wireless mesh networks. Computers \& Electrical Engineering 2016;56:113-29. DOI

36. Moad S, Hansen MT, Jurdak R, Kusy B, Bouabdallah N. WETX: A weighted expected transmission routing metric for diversity in wireless sensor networks. Proceedings of the 2011 IFIP Wireless Days (WD); 2011 Oct 10-12; Niagara Falls, ON, Canada. New York: IEEE; 2011. p. 1-7. DOI

37. Kum D, Le A, Cho Y, Toh CK, Lee I. An efficient on-demand routing approach with directional flooding for wireless mesh networks. J Commun Netw 2010;12:67-73. DOI

38. Zhao L, Yu Z, Niu J, Zhang H, Ding W. A hybrid routing protocol for hierarchy wireless mesh networks. Proceedings of the 6th International Conference on Wireless Communications Networking and Mobile Computing (WiCOM); 2010 Sep 23-25; Chengdu, China. New York: IEEE; 2010. p. 1-4. DOI

39. Clausen T, Jacquet P. Optimized Link State Routing Protocol (OLSR). 2003. Available from: https://tools.ietf.org/html/rfc3626. [Last accessed on 31 Mar 2021].

40. Perkins C, Belding-Royer E, Das S. Ad Hoc On-demand Distance Vector (Aodv) Routing. 2003. Available from: https://tools.ietf.org/html/rfc3561. [Last accessed on 31 Mar 2021].

41. Le A, Kum D, Cho Y. An efficient hybrid routing approach for hybrid wireless mesh networks. In: Park JH, Chen H, Atiquzzaman M, Lee C, Kim T, Yeo S, editors. Advances in Information Security and Assurance. Berlin: Springer Berlin Heidelberg; 2009. p. 53242.

42. Chai Y, Shi W, Shi T, Yang X. An efficient cooperative hybrid routing protocol for hybrid wireless mesh networks. Wireless Netw 2017;23:1387-99. DOI

43. Chai Y, Shi W, Shi T. Load-aware cooperative hybrid routing protocol in hybrid wireless mesh networks. Int J Electron Commun 2017;74:135-44. DOI

44. Chai Y, Zeng X. Regional condition-aware hybrid routing protocol for hybrid wireless mesh network. Comput Netw 2019;148:120-8. 
DOI

45. Bao X, Tan W, Nie J, Lu C, Jin G. Design of logical topology with K-connected constraints and channel assignment for multi-radio wireless mesh networks. Int J Commun Syst 2017;30:e2914. DOI

46. Ali S, Ngadi MA. Optimized interference aware joint channel assignment model for wireless mesh network. Telecommun Syst 2016;62:215-30. DOI

47. Tang J, Xue G, Zhang W. Cross-layer design for end-to-end throughput and fairness enhancement in multi-channel wireless mesh networks. IEEE Trans Wireless Commun 2007;6:3482-6. DOI

48. Avallone $\mathrm{S}$, Banchs A. A channel assignment and routing algorithm for energy harvesting multiradio wireless mesh networks. IEEE $J$ Select Areas Commun 2016;34:1463-76. DOI

49. Zhang D, Zhu Y, Liu S, Zhang X, Song J. Multi-radio multi-channel (MRMC) resource optimization method for wireless mesh network. Journal of Information Science and Engineering 2016;32:495-514. DOI

50. Deng X, Luo J, He L, Liu Q, Li X, Cai L. Cooperative channel allocation and scheduling in multi-interface wireless mesh networks. Peer-to-Peer Netw Appl 2019;12:1-12. DOI

51. Sahni Y, Cao J, Zhang S, Yang L. Edge Mesh: A new paradigm to enable distributed intelligence in internet of things. IEEE Access 2017;5:16441-58. DOI

52. Liu E, Deng X, Cao Z, Zhang H. Design and evaluation of a prediction-based dynamic edge computing system. Proceedings of the 2018 IEEE Global Communications Conference (GLOBECOM); 2018 Dec 9-13; Abu Dhabi, United Arab Emirates. New York: IEEE; 2018. p. 1-6. DOI

53. Shi W, Zhang J, Zhang R, Hu K. An area-based offloading policy for computing offloading in mec-assisted wireless mesh network. Proceedings of the 2019 IEEE/CIC International Conference on Communications in China (ICCC); 2019 Aug 11-13; Changchun, China. New York: IEEE; 2019. p. 507-11. DOI

54. Ramakrishnan B, Selvi M, Nishanth RB. Efficiency measure of routing protocols in vehicular ad hoc network using freeway mobility model. Wireless Netw 2017;23:323-33. DOI

55. Acharjee T, Roy S. A mobility-aware cluster based routing for large wireless mesh network. Proceedings of the 2016 International Conference on Signal Processing, Communication, Power and Embedded System (SCOPES); 2016 Oct 3-5; Paralakhemundi. New York: IEEE; 2016. p. 1376-80. DOI

56. Rajesh MV, Gireendranath TVS, Murthy JVR. A novel energy efficient cluster based routing protocol for highly dense MANET architecture. IJCIR 2017;13:719-44. Available from: http://www.ripublication.com/ijcir17/ijcirv13n5_06.pdf. [Last accessed on 22 Mar 2021]

57. Acharjee T, Rajkonwar S, Roy S. An improved hierarchical cluster based routing approach for wireless mesh network. Proceedings of the 2016 International Conference on Computer Communication and Informatics (ICCCI); 2016 Jan 7-9; Coimbatore, India. New York: IEEE; 2016. p. 1-6. DOI

58. Prakash SPS, Nagabhushan TN, Krinkin K. Cluster based approach to minimize delay in energy aware routing for ieee $802.11 \mathrm{~s}$ Wireless Mesh Networks under mobility conditions. Proceedings of the 2016 19th Conference of Open Innovations Association (FRUCT); 2016 Nov 7-11; Jyvaskyla. New York: IEEE; 2016. p. 189-95. DOI

59. Cheng H, Yang S, Cao J. Dynamic genetic algorithms for the dynamic load balanced clustering problem in mobile ad hoc networks. Expert Systems with Applications 2013;40:1381-92. DOI

60. Sgora A, Vergados DD, Chatzimisios P. A survey on security and privacy issues in wireless mesh networks. Secur Commun Netw 2016;9:1877-89. DOI

61. Zhang Y, Luo J, Hu H. Wireless mesh networking: architectures, protocols and standards. 1st ed. Hoboken, NJ: Taylor and Francis; 2007. p. 610. Available from: https://www.routledge.com/Wireless-Mesh-Networking-Architectures-Protocols-and-Standards/ZhangLuo-Hu/p/book/9780849373992. [Last accessed on 22 Mar 2021].

62. Venkataraman R, Pushpalatha M, Rao TR. Performance analysis of flooding attack prevention algorithm in MANETs. International Journal of Computer and Information Engineering 2009;3:2056-9. DOI

63. Winjum E, Hegland AM, Kure Ø, Spilling P. Replay Attacks in Mobile Wireless Ad Hoc Networks: Protecting the OLSR Protocol. In: Lorenz P, Dini P, editors. Networking - ICN 2005. Berlin: Springer Berlin Heidelberg; 2005. pp. 471-9. DOI

64. Shaikh FA, Patil U. Efficient detection and prevention of wormhole attacks in wireless mesh network. IRJET 2017;4:2208-14. Available from: https://www.irjet.net/archives/V4/i5/IRJET-V4I5578.pdf. [Last accessed on 22 Mar 2021]

65. Yu Y, Ning Z, Guo L. A secure routing scheme based on social network analysis in wireless mesh networks. Sci China Inf Sci 2016;59:122310. DOI

66. Pugalendhi G, Velusamy D, Paul A, Kim K. Fuzzy-based trusted routing to mitigate packet dropping attack between data aggregation points in smart grid communication network. Computing 2017;99:81-106. DOI

67. Shiva S, Nagabhushan T, Krinkin K. Energy Aware Power Save Mode based AODV Routing for IEEE 802.11s Wireless Mesh Networks. IJCA 2016;137:1-7. DOI

68. de Figueiredo Marques V, Kniess J, Parpinelli RS. An energy efficient mesh LNN routing protocol based on ant colony optimization. Proceedings of the 2018 IEEE 16th International Conference on Industrial Informatics (INDIN); 2018 Jul 18-20; Porto, Portugal. New York: IEEE; 2018. p. 43-8. DOI

69. Chettibi S, Chikhi S. Adaptive maximum-lifetime routing in mobile ad-hoc networks using temporal difference reinforcement learning. Evolving Systems 2014;5:89-108. DOI

70. Guo W, Yan C, Lu T. Optimizing the lifetime of wireless sensor networks via reinforcement-learning-based routing. Int $J$ Distrib Sens Netw 2019;15:155014771983354. DOI 
71. Kassan S, Gaber J, Lorenz P. Game theory based distributed clustering approach to maximize wireless sensors network lifetime. J Net Comput Appl 2018;123:80-8. DOI

72. Wu Y, Yang K. Cooperative reinforcement learning based throughput optimization in energy harvesting wireless sensor networks. Proceedings of the 2018 27th Wireless and Optical Communication Conference (WOCC); 2018 Apr 30-May 1; Hualien, Taiwan. New York: IEEE; 2018. p. 1-6. DOI

73. Maleki M, Hakami V, Dehghan M. A model-based reinforcement learning algorithm for routing in energy harvesting mobile ad-hoc networks. Wireless Pers Commun 2017;95:3119-39. DOI

74. Dong Y, Wang J, Shim B, Kim DI. DEARER: A distance-and-energy-aware routing with energy reservation for energy harvesting wireless sensor networks. IEEE J Select Areas Commun 2016;34:3798-813. DOI

75. Chettibi S, Chikhi S. Dynamic fuzzy logic and reinforcement learning for adaptive energy efficient routing in mobile ad-hoc networks. Applied Soft Computing 2016;38:321-8. DOI

76. Yao Q, Huang A, Shan H, Quek TQS, Wang W. Delay-aware wireless powered communication networks-energy balancing and optimization. IEEE Trans Wireless Commun 2016;15:5272-86. DOI

77. Suto K, Nishiyama H, Kato N, Kuri T. Model predictive joint transmit power control for improving system availability in energyharvesting wireless mesh networks. IEEE Commun Lett 2018;22:2112-5. DOI

78. Yan L, Agrell E, Dharmaweera MN, Wymeersch H. Joint assignment of power, routing, and spectrum in static flexible-grid networks. J Lightwave Technol 2017;35:1766-74. DOI

79. Liu Y, Tong KF, Qiu X, Liu Y, Ding X. Wireless mesh networks in IoT networks. Proceedings of the 2017 International Workshop on Electromagnetics: Applications and Student Innovation Competition; 2017 May 30-Jun 1; London, UK. New York: IEEE; 2017. p. 183-5. DOI

80. Karunaratne S, Gacanin H. An overview of machine learning approaches in wireless mesh networks. IEEE Commun Mag 2019;57:102-8. DOI

81. Bote-lorenzo ML, Gómez-sánchez E, Mediavilla-pastor C, Asensio-pérez JI. Online machine learning algorithms to predict link quality in community wireless mesh networks. Computer Networks 2018;132:68-80. DOI

82. Sakib S, Tazrin T, Fouda MM, Fadlullah ZM, Nasser N. A deep learning method for predictive channel assignment in beyond $5 \mathrm{G}$ networks. IEEE Network 2021;35:266-72. DOI

83. Van Moffaert K, Drugan MM, Nowé A. Scalarized multi-objective reinforcement learning: Novel design techniques. Proceedings of the 2013 IEEE Symposium on Adaptive Dynamic Programming and Reinforcement Learning (ADPRL); 2013 Apr 16-19; Singapore. New York: IEEE; 2013. p. 191-9. DOI

84. Al-saadi A, Setchi R, Hicks Y, Allen SM. Routing protocol for heterogeneous wireless mesh networks. IEEE Trans Veh Technol 2016;65:9773-86. DOI

85. Farahnakian F, Ebrahimi M, Daneshtalab M, Liljeberg P, Plosila J. Q-learning based congestion-aware routing algorithm for on-chip network. Proceedings of the 2011 IEEE 2nd International Conference on Networked Embedded Systems for Enterprise Applications; 2011 Dec 8-9; Perth, WA, Australia. New York: IEEE; 2011. p. 1-7. DOI

86. Dong S, Agrawal P, Sivalingam K. Reinforcement learning based geographic routing protocol for UWB wireless sensor network. Proceedings of the IEEE GLOBECOM 2007-IEEE Global Telecommunications Conference; 2007 Nov 26-30; Washington, DC, USA. New York: IEEE; 2007. p. 652-6. DOI

87. Lee J, Kang J; Ajou University; Korea. Interference and Mobility-Aware Routing Scheme Based on Reinforcement Learning. IJFCC 2016;5:94-8. DOI

88. Coutinho N, Matos R, Marques C, et al. Dynamic dual-reinforcement-learning routing strategies for quality of experience-aware wireless mesh networking. Computer Networks 2015;88:269-85. DOI

89. Tang F, Mao B, Fadlullah ZM, et al. On removing routing protocol from future wireless networks: A real-time deep learning approach for intelligent traffic control. IEEE Wireless Commun 2018;25:154-60. DOI

90. Mao B, Fadlullah ZM, Tang F, et al. Routing or computing? IEEE Trans Comput 2017;66:1946-60. DOI

91. Chinchali S, Hu P, Chu T, et al. Cellular network traffic scheduling with deep reinforcement learning. Available from: https://stanfordasl.github.io/wp-content/papercite-data/pdf/Chinchali.ea.AAAI18.pdf. [Last accessed on 22 Mar 2021].

92. Stampa G, Arias M, Sánchez-Charles D, Muntés-Mulero V, Cabellos A. A deep-reinforcement learning approach for softwaredefined networking routing optimization. Available from: https://arxiv.org/abs/1709.07080. [Last accessed on 22 Mar 2021].

93. Dugaev DA, Matveev IG, Siemens E, Shuvalov VP. Adaptive reinforcement learning-based routing protocol for wireless multihop networks. Proceedings of the 2018 XIV International Scientific-Technical Conference on Actual Problems of Electronics Instrument Engineering (APEIE); 2018 Oct 2-6; Novosibirsk, Russia. New York: IEEE; 2018. p. 209-18. DOI

94. Sendra S, Rego A, Lloret J, Jimenez JM, Romero O. Including artificial intelligence in a routing protocol using software defined networks. Proceedings of the 2017 IEEE International Conference on Communications Workshops (ICC Workshops); 2017 May 125; Paris, France. New York: IEEE; 2017. p. 670-4. DOI

95. Xu H, Li X, Huang L, Deng H, Huang H, Wang H. Incremental deployment and throughput maximization routing for a hybrid SDN. IEEE/ACM Trans Networking 2017;25:1861-75. DOI

96. Peng Y, Guo L, Deng Q, Ning Z, Zhang L. A novel hybrid routing forwarding algorithm in SDN enabled wireless mesh networks. Proceedings of the IEEE 17th International Conference on High Performance Computing and Communications, 2015 IEEE 7th International Symposium on Cyberspace Safety and Security, and 2015 IEEE 12th International Conference on Embedded Software and Systems; 2015 Aug 24-26; New York, NY, USA. New York: IEEE; 2015. p. 1806-11. DOI

97. Bano M, Gilani SSA, Qayyum A. A comparative analysis of hybrid routing schemes for SDN based wireless mesh networks. 
Proceedings of the 2018 IEEE 20th International Conference on High Performance Computing and Communications; IEEE 16th International Conference on Smart City; IEEE 4th International Conference on Data Science and Systems (HPCC/SmartCity/DSS); 2018 Jun 28-30; Exeter, UK. New York: IEEE; 2018. p. 1189-94. DOI

98. Huang H, Li P, Guo S, Zhuang W. Software-defined wireless mesh networks: architecture and traffic orchestration. IEEE Network 2015;29:24-30. DOI

99. Labraoui M, Boc MM, Fladenmuller A. Software defined networking-assisted routing in wireless mesh networks. Proceedings of the 2016 International Wireless Communications and Mobile Computing Conference (IWCMC); 2016 Sep 5-9; Paphos, Cyprus. New York: IEEE; 2016. p. 377-82. DOI

100. Detti A, Pisa C, Salsano S, Blefari-Melazzi N. Wireless mesh software defined networks (wmSDN). Proceedings of the 2013 IEEE 9th international conference on wireless and mobile computing, networking and communications (WiMob); 2013 Oct 7-9; Lyon, France. New York: IEEE; 2013. p. 89-95. DOI

101. Ashraf U. Energy-aware gateway placement in green wireless mesh networks. IEEE Commun Lett 2017;21:156-9. DOI

102. Bello OM, Taiwe KD. Mesh node placement in wireless mesh network based on multiobjective evolutionary metaheuristic. International Journal of Autonomic Computing 2017;2:231-54. DOI

103. Xu C, Wang K, Li P, Xia R, Guo S, Guo M. Renewable energy-aware big data analytics in geo-distributed data centers with reinforcement learning. IEEE Trans Netw Sci Eng 2020;7:205-15. DOI

104. Fadlullah ZM, Nakajo T, Nishiyama H, Owada Y, Hamaguchi K, Kato N. Field measurement of an implemented solar powered BSbased wireless mesh network. IEEE Wireless Commun 2015;22:137-43. DOI 